\title{
Visualización del Plano Oclusal y Guía Anterior en Desdentados Parciales en la Telerradiografía Lateral: Descripción de la Técnica a Través de Casos Clínicos
}

\author{
Occlusal Plane and Anterior Guidance Visualization in Lateral Cephalometric Radiographs \\ in Partially Edentulous Patients: Technique Description through Case Reports
}

\author{
Rolando Schulz Rosales*; Bárbara Cerda Peralta*; Milena Moya Munjin ${ }^{* * *}$ \& Fernando Romo Ormazábal ${ }^{\star * * *}$
}

SCHULZ, R. R.; CERDA, P. B.; MOYA, M. M. \& ROMO, O. F. Visualización del plano oclusal y guía anterior en desdentados parciales en la telerradiografía lateral: Descripción de la técnica a través de casos clínicos. Int. J. Odontostomat., 7(3):465-470, 2013.

RESUMEN: El uso de la telerradiografía lateral ha tomado gran importancia en Rehabilitación Oral ayudando al clínico en el diagnóstico y planificación del tratamiento a través de la identificación del biotipo facial del paciente, la inclinación del plano oclusal, la relación anteroposterior entre maxilar y mandíbula, inclinación de los ejes dentarios y como ayuda complementaria en casos de alteraciones de la Dimensión Vertical. El problema surge cuando estamos frente a pacientes desdentados que han perdido puntos de referencia cefalométricos necesarios para el análisis. El presente trabajo muestra una técnica para poder visualizar radiográficamente el plano oclusal y la guía anterior utilizando láminas de plomo.

PALABRAS CLAVE: análisis cefalométrico, telerradiografía lateral, desdentado parcial, plano oclusal, guía anterior, dimensión vertical.

\section{INTRODUCCIÓN}

La cefalometría es una herramienta fundamental para el diagnóstico y control del tratamiento en Ortodoncia, sin embargo, aun cuando los ortodoncistas la utilizan en forma rutinaria desde hace más de siete décadas, los rehabilitadores orales aún no la incorporan masivamente a su práctica clínica.

El análisis cefalométrico se basa en la identificación de puntos de reparo anatómicos y planos de referencia radiográficos, con el fin de realizar mediciones lineales, angulares y proporciones. Sin embargo, hay que considerar que muchas de estas referencias se encuentran en los dientes, que pueden estar ausentes en el desdentado parcial. Asimismo, en un paciente desdentado la mandíbula cambia su posición espacial y por tanto se alteran sus relaciones con las estructuras craneales y dentarias.
Dentro de los aportes de la cefalometría en Rehabilitación Oral se encuentran: la identificación del biotipo facial del paciente, la inclinación del plano oclusal, la relación anteroposterior de los maxilares, inclinación de los ejes dentarios, como ayuda complementaria en casos de alteraciones de la Dimensión Vertical y en la estética facial (Strajnic et al., 2008).

El biotipo corresponde a un conjunto de rasgos biológicos que caracterizan a individuos de la misma especie, siendo variaciones pequeñas, siempre dentro de rangos normales. La identificación del biotipo es una ayuda al momento de la selección del largo de los dientes, altura de cúspides, guías de desoclusión y ancho del arco dentario.

La determinación del plano oclusal es crucial al momento de planificar un tratamiento, determinando el

* Profesor Asistente, Facultad de Odontología, Universidad de Chile, Clínica Integral del Adulto, Santiago, Chile.

** Instructor, Facultad de Odontología, Universidad de Chile, Clínica Integral del Adulto, Santiago, Chile.

*** Ayudante, Facultad de Odontología, Universidad de Chile, Clínica Integral del Adulto, Santiago, Chile.

**** Profesor Titular, Facultad de Odontología, Universidad de Chile, Departamento de Prótesis, Santiago, Chile. 
espacio protético disponible, la estética, la altura de las cúspides y las guías de desoclusión para nuestra rehabilitación. Cefalométricamente, Ricketts define el plano oclusal como la línea que toca las puntas de cúspides de molares y premolares inferiores. En pacientes desdentados esta referencia está alterada o perdida y debemos acudir a los rodetes de altura para recuperarla.

Por su parte la inclinación de los ejes dentarios es fundamental en la estética y la función oclusal. La determinación de la posición de los incisivos y la guía anterior son pilares esenciales en la rehabilitación protésica (Bloom \& Padayachy, 2006). La cefalometría es una herramienta que tiene la misma importancia que el encerado diagnóstico, puesto que permite al clínico tomar decisiones relevantes tales como cambios de ejes dentarios, desvitalización de dientes, exodoncias y cambios de dimensión vertical. Otro aspecto importante que permite la cefalometría es observar las relaciones sagitales entre las bases óseas maxilares y ver si éstas se encuentran en armonía para sustentar la futura rehabilitación o si se debe hacer modificaciones en la planificación.

En relación a la dimensión vertical se sabe que hay múltiples métodos de obtención de ésta, existiendo métodos subjetivos (Fonético, deglución, posición de reposo mandibular, registros pre-extracción) y métodos objetivos, entre los cuales encontramos, Índice de Willis, mediciones faciales, craneométricos y cefalométricos (Misch, 2000).

La altura facial inferior es un concepto cefalométrico que se corresponde con el concepto clínico de dimensión vertical. La dimensión vertical se define como la distancia entre cualquier punto del tercio medio y otro del tercio inferior del rostro medida en milímetros, en el plano frontal en piel (The Glossary of Prosthodontic Terms, 2005). Debemos diferenciar entre dimensión vertical postural (DVP) y dimensión vertical oclusal (DVO). La DVP es la altura del tercio inferior cuando la mandíbula se encuentra en posición de reposo, cuando la musculatura está en equilibrio tónico y los arcos se encuentran ligeramente en inoclusión. Por su parte la DVO es aquella medida cuando los dientes se encuentran en máxima intercuspidación (Manns \& Biotti, 2006). La estética facial está íntimamente relacionada con el soporte de los labios y la DVO. Una creencia común entre los rehabilitadores orales es que un aumento o disminución de la DVO afecta significativamente la altura facial inferior y la estética facial (Gross et al., 2002).

Una limitación importante al momento de hacer el análisis cefalométrico en pacientes desdentados es que muchos puntos anatómicos se encuentran en los dientes, y cuando éstos se pierden, el clínico tiene problemas para determinar la posición en que debieran ir. En los desdentados totales la telerradiografía habitualmente se toma en posición de reposo, lo que no tiene valor diagnóstico ya que las normas cefalométricas de todos los análisis están concebidas en máxima intercuspidación. Además es una posición que tiene múltiples variaciones a lo largo del tiempo (Miralles et al., 2001). En los portadores de prótesis la radiolucidez del acrílico impide ubicar estructuras como el plano oclusal o los dientes protésicos en la telerradiografía. Es por esto que proponemos la utilización de rodetes de altura y/o prótesis de trabajo para la toma radiográfica, restableciendo el plano oclusal y brindando estabilidad mandibular al paciente; a éstas se adiciona un material radiopaco para visualizar las estructuras de referencia y poder realizar el trazado cefalométrico.

Descripción de la técnica. Existen varios materiales radiopacos posibles de utilizar: Plomo, Gutapercha, Alambres, Sulfato de bario, Esferas metálicas, Resinas Compuestas radiopacas. Las láminas de plomo se obtienen fácilmente de las películas radiográficas intraorales, son maleables y tienen alta radiopacidad. Nuestra experiencia utilizando dichas láminas ha sido muy buena, por lo que recomendamos su utilización.

Los rodetes de altura deben ser confeccionados con cera sobre una base de acrílico, evitando utilizar laca base por su fragilidad y posible deformación. Deben tener las mismas características y/o requisitos que las prótesis en cuanto a: Determinación del plano oclusal, contorno de los dientes, soporte y cierre labial, overjet, overbite, línea media dentaria, línea blanca, línea de la sonrisa, triángulos negros, etc.

Una vez confeccionado el rodete de altura se procede a pegar mediante goteo de cera una lámina de plomo de tres capas de grosor, $30 \mathrm{~mm}$ de largo y $4 \mathrm{~mm}$ de ancho. Si el paciente cuenta con una prótesis antigua o de trabajo se adhiere la lámina mediante adhesivo de cianoacrilato u otro adhesivo a elección. Las láminas de plomo se adhieren a lugares estratégicos, como son el plano oclusal y en el contorno anterior en la línea media.

Con el rodete o prótesis en boca y con las láminas de plomo adheridas a ellas, el paciente puede ser sometido a la toma radiográfica. La posición de oclusión es determinada por el clínico pero nunca debe ser en posición postural de reposo clínico. Por otra parte se sugiere entrenar al paciente en la posición en que se desea tomar la radiografía y en lo posible acompañarlo 
durante la toma radiográfica. Se puede utilizar adhesivo de prótesis o acondicionador de tejidos en los casos que las prótesis o rodetes se encuentren inestables.

A continuación mediante 4 casos clínicos se explicará la técnica propuesta para visualizar el plano oclusal y la guía anterior en una telerradiografía de perfil en desdentados parciales.

Caso 1. Se presenta una mujer de 53 años de edad con destrucción coronaria completa y periodontitis crónica avanzada. Se han perdido los puntos de referencia cefalométricos de los incisivos superiores. Previo a las extracciones dentarias se confecciona una placa de altura determinando plano oclusal, dimensión vertical, parámetros estéticos, contorno de los dientes anteriores, overjet y overbite y posición inicial de tratamiento. Posterior a eso se fijan con cera láminas de plomo en la línea media superior y a nivel del plano oclusal previo a la toma radiográfica. Durante el momento de la toma radiográfica el paciente se encuentra en MIC. En la radiografía obtenida se puede apreciar entonces claramente el contorno dentario y plano oclusal; esto permite ubicar los puntos para realizar un análisis cefalométrico preciso y reproducible (Fig. 1).

Caso 2. Hombre de 55 años, desdentado parcial no portador de prótesis. Presenta ausencia de incisivos superiores e inferiores, migración dentaria patológica (Brunsvold, 2005), mordida abierta anterior e interposición lingual. Se realiza un primer montaje para definir la posición inicial de tratamiento considerando parámetros estéticos y de soporte labial, se decidió mantener la mordida abierta anterior para lograr una estética anterior aceptable. Luego se toma telerradiografía lateral con láminas de plomo en ambos arcos y en la zona de línea media superior e inferior, con el paciente ocluyendo, y ahora es posible realizar un trazado cefalométrico con los puntos de referencia dentarios localizados en forma precisa (Fig. 2).

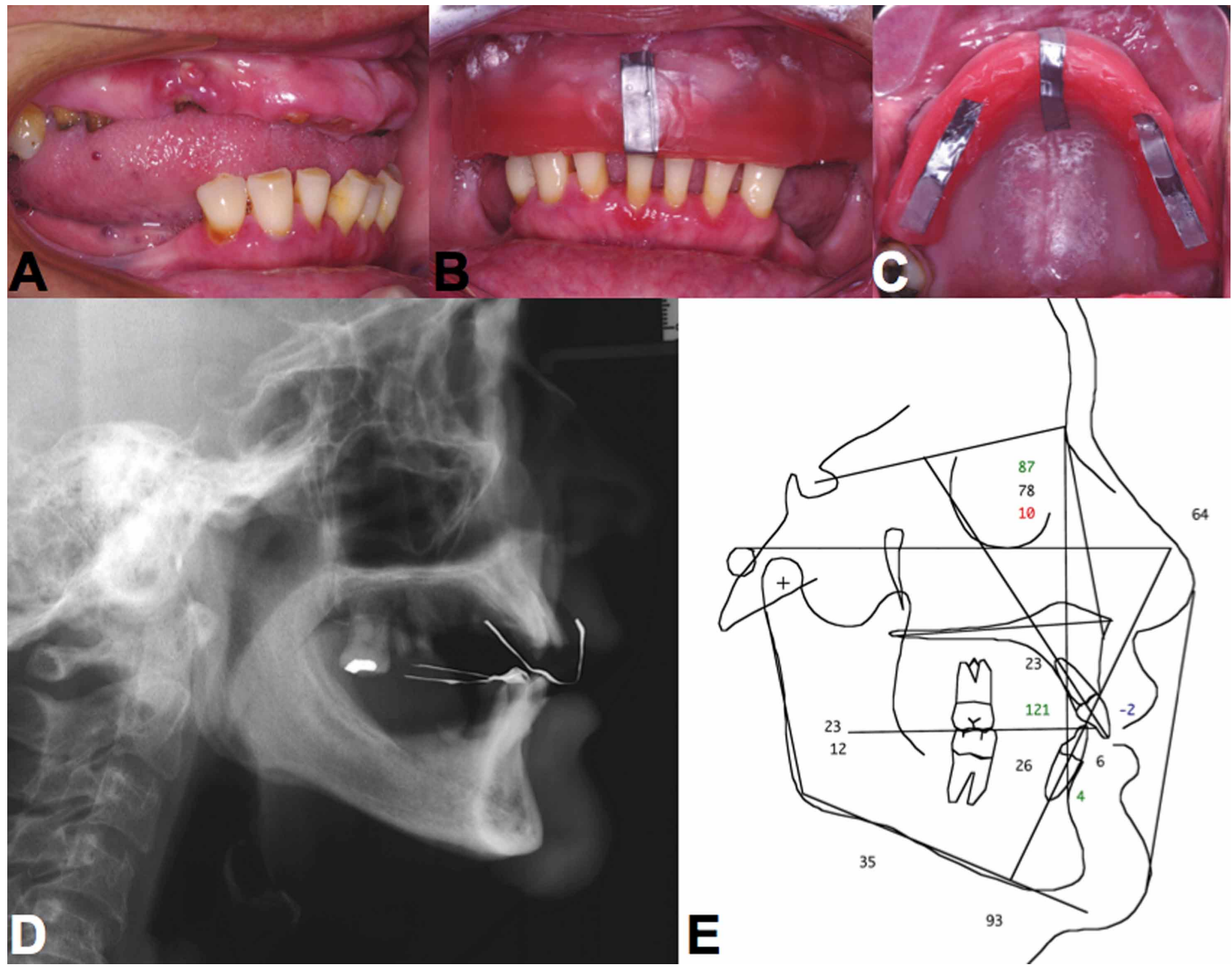

Fig. 1. Mujer de 53 años de edad con destrucción coronaria completa y periodontitis crónica avanzada. 

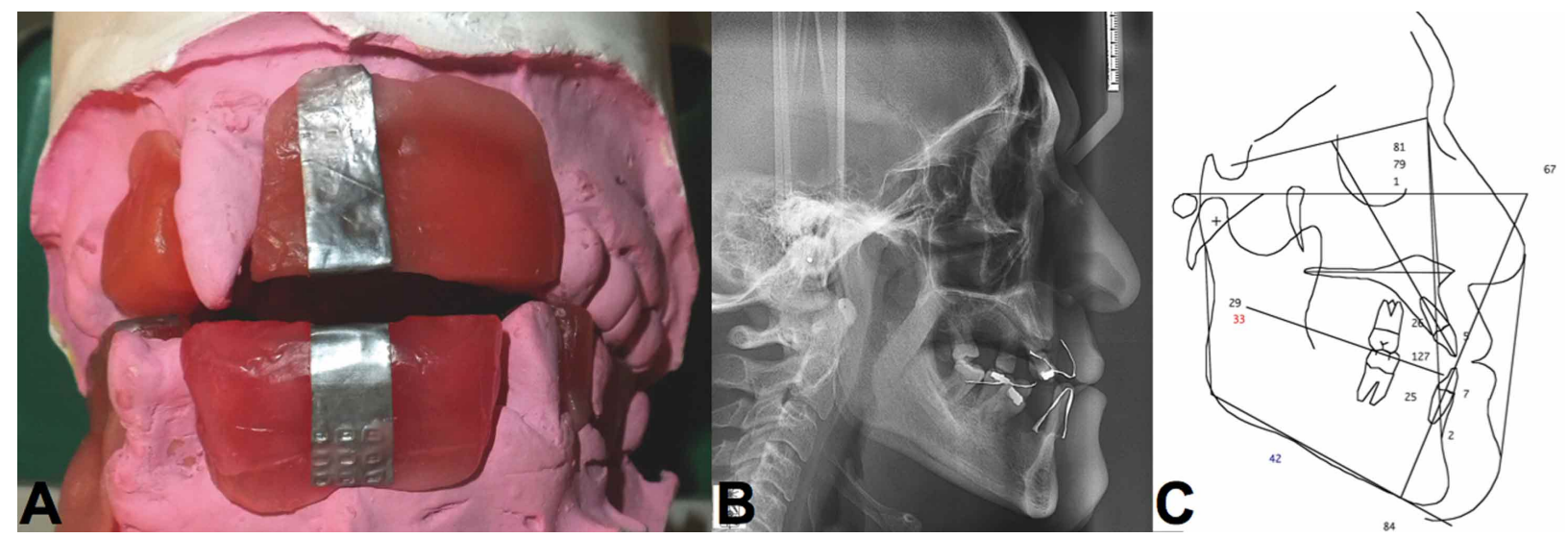

Fig. 2. Hombre de 55 años, desdentado parcial no portador de prótesis.

Caso 3. Mujer de 66 años, desdentada parcial portadora de prótesis acrílicas, en la cual se desea investigar si existe pérdida de dimensión vertical oclusal. Las prótesis acrílicas son radiolúcidas, por lo que se procede a pegar láminas de plomo sobre las prótesis con adhesivo de cianoacrilato, en la zona anterior y a nivel del plano oclusal (Fig. 3).

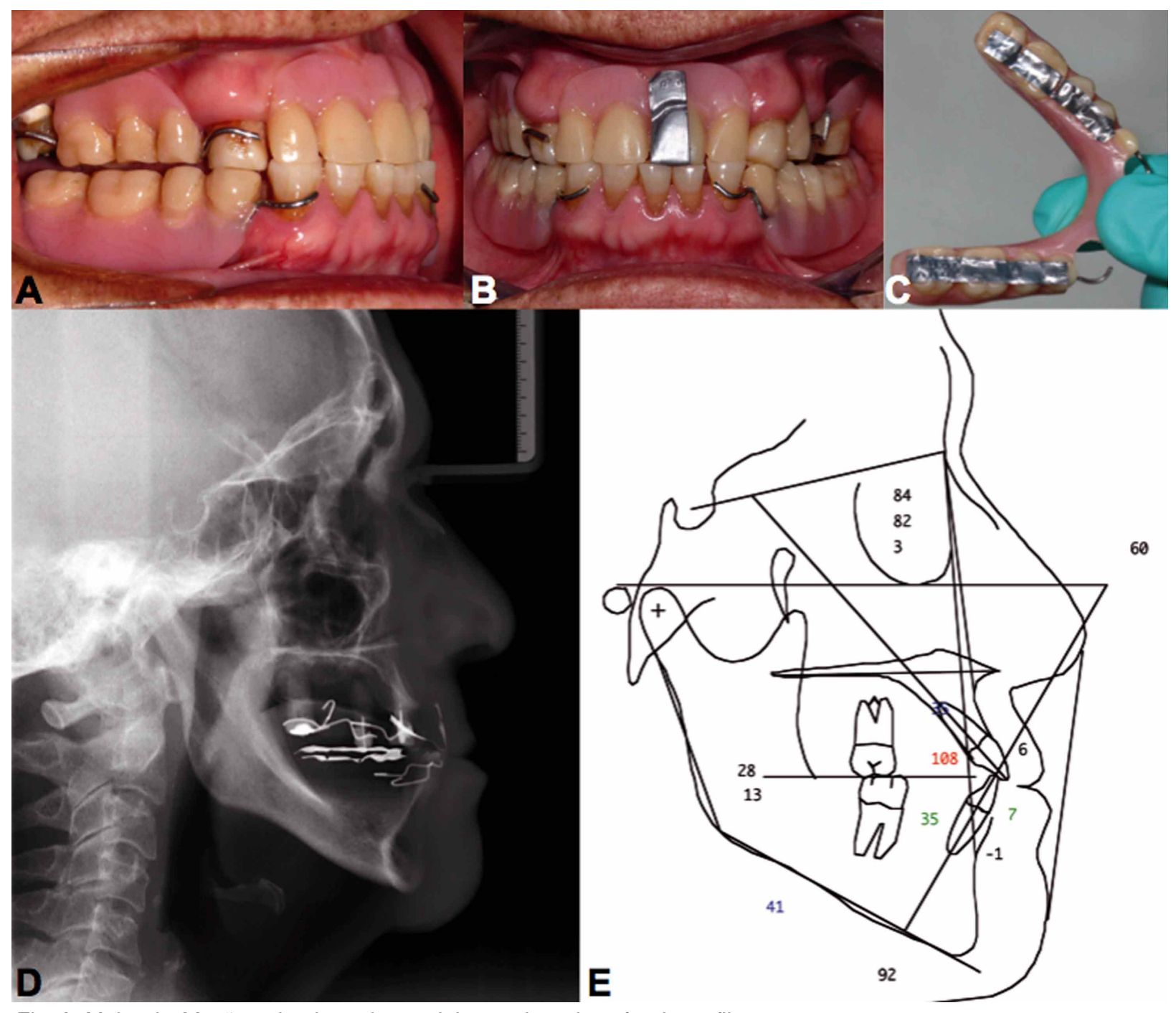

Fig. 3. Mujer de 66 años, desdentada parcial portadora de prótesis acrílicas. 
Caso 4. Mujer de 70 años rehabilitada con implantes y sobredentadura. Al igual que en el caso anterior la radiografía se toma con prótesis en oclusión y la lámi- na de plomo permite visualizar el contorno de los incisivos (Fig. 4).

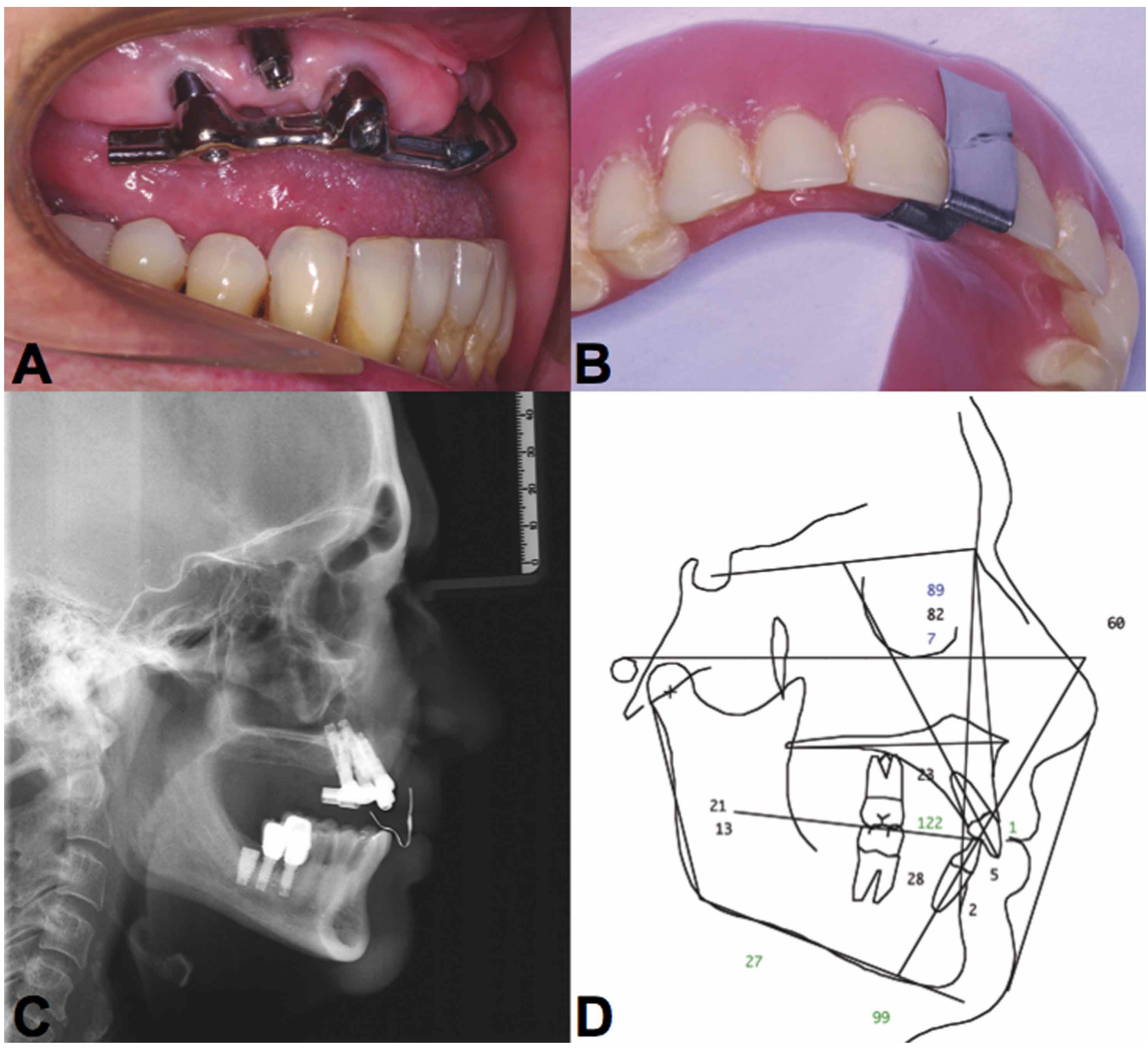

Fig. 4. Mujer de 70 años rehabilitada con implantes y sobredentadura.

\section{DISCUSIÓN}

En la revisión de literatura algunos autores mencionan la visualización del plano oclusal y el eje de los incisivos en la radiografía con diferentes técnicas y materiales, Jyothi et al. (2012), proponen como una posible solución utilizar alambres contorneados adaptados sobre las prótesis, pero tiene la dificultad de la fijación del alambre en posición y lo difícil que es contornearlo. Bassi et al. (2001) mencionan la utiliza- ción de láminas de plomo para visualizar el contorno de incisivos inferiores en prótesis completas, pero en su publicación no dan mayores detalles al respecto. Brzoza et al. (2005) insertaron conos de gutapercha dentro de los rodetes con el mismo objetivo, aunque al igual que los demás no muestran fotografías ni se explica la técnica en detalle. Por su parte Jayachandran et al. (2007), utiliza esferas metálicas adheridas a la 
base acrílica de una prótesis de trabajo y a nivel del plano oclusal una platina metálica adherida con cera. Su limitación es que solo puede ser utilizada en desdentados totales ya que la irregularidad del plano oclusal en desdentados parciales impediría el uso de la platina y en ocasiones no existirían los espacios para la colocación de las esferas.

A través de los casos anteriormente presentados se puede resaltar que la utilidad de la telerradiografía lateral y el análisis cefalométrico se da en todas las eta- pas del tratamiento rehabilitador. Se puede utilizar en el momento del diagnóstico, en las etapas de prueba en cera de las futuras prótesis y también en los casos terminados para la confirmación del plano oclusal, de la posición de los incisivos, y la dimensión vertical oclusal.

Finalmente a través de una técnica simple, reversible, que no requiere de mayor tiempo ni recursos clínicos, podemos devolver las referencias anatómicas perdidas necesarias para realizar un análisis cefelométrico fidedigno.

SCHULZ, R. R.; CERDA, P. B.; MOYA, M. M. \& ROMO, O. F. Occlusal plane and anterior guidance visualization in lateral cephalometric radiographs in partially edentulous patients: Technique description through case reports. Int. J. Odontostomat., 7(3):465-470, 2013.

ABSTRACT: The use of lateral cephalometric radiographs has become very important in Oral Rehabilitation, helping the clinician in the diagnosis and treatment planning through the identification of facial biotype, occlusal plane inclination, sagittal relationship between maxilla and mandible, inclination of the dental axes, and a valuable help in assessing alterations of the vertical dimension of occlusion. The problem arises when treating partially edentulous patients who have lost their cephalometric reference points necessary for the analysis. This paper shows a technique to visualize the occlusal plane and anterior guidance in partially edentulous patients who have lost these anatomical landmarks in a lateral cephalometric radiograph, using lead sheets.

KEY WORDS: cephalometric analysis, partial dentures, edentulous, occlusal plane, anterior guidance, vertical dimension.

\section{REFERENCIAS BIBLIOGRÁFICAS}

Bassi, F.; Rizzatti, A.; Schierano, G. \& Preti, G. Evaluation of the utility of cephalometric parameters in constructing complete denture. Part II: placement of anterior teeth. J. Oral Rehabil., 28(4):349-53, 2001.

Bloom, D. R. \& Padayachy, J. Increasing occlusal vertical dimension--why, when and how. Br. Dent. J., 200(5):2516, 2006.

Brunsvold, M. A. Pathologic tooth migration. J. Periodontol., 76(6):859-66, 2005.

Brzoza, D.; Barrera, N.; Contasti, G. \& Hernández, A. Predicting vertical dimension with cephalograms, for edentulous patients. Gerodontology, 22(2):98-103, 2005.

Gross, M. D.; Nissan, J.; Ormianer, Z.; Dvori, S. \& Shifman, A. The effect of increasing occlusal vertical dimension on face height. Int. J. Prosthodont., 15(4):353-7, 2002.

Jayachandran, S.; Ramachandran, C. R. \& Varghese, R. Occlusal plane orientation: a statistical and clinical analysis in different clinical situations. J. Prosthodont., 17(7):572-5, 2008.

Jyothi, P. A.; Poojya, R. \& Shruthi, C. S. Bioscopic planes of prosthetic interest to establish occlusal plane in edentulous patients-A cephalometric study. Ann. Essences Dent., 4(1):38-42, 2012.
Manns, A. \& Biotti, J. Manual práctico de oclusión dental. 2a ed. Caracas, Amolca, 2006.

Miralles, R.; Dodds, C.; Palazzi, C.; Jaramillo, C.; Quezada, V.; Ormeño, G. \& Villegas, R. Vertical dimension. Part 1: comparison of clinical freeway space. Cranio, 19(4):2306, 2001.

Misch, C. E. Clinical indications for altering vertical dimension of occlusion. Objective vs subjective methods for determining vertical dimension of occlusion. Quintessence Int., 31(4):280-2, 2000.

Strajnic, L.; Stanisic-Sinobad, D.; Markovic, D. \& Stojanovic, L. Cephalometric indicators of the vertical dimension of occlusion. Coll. Antropol., 32(2):535-41, 2008.

The glossary of prosthodontic terms. J. Prosthet. Dent., 94(1):10-92, 2005.

Dirección para Correspondencia:

Dr. Rolando Schulz Rosales

Profesor asistente

Clínica Integral del Adulto

Facultad de odontología

Universidad de Chile

Santiago

CHILE

Recibido : 23-04-2013

Email: schulzrolando@gmail.com
Aceptado: 03-08-2013 